\title{
Do Multi-hop Readers Dream of Reasoning Chains?
}

\author{
Haoyu Wang * ${ }^{*} \quad$ Mo Yu ${ }^{* \dagger} \quad$ Xiaoxiao Guo ${ }^{* \dagger} \quad$ Rajarshi Das ${ }^{* \dagger}$ \\ Wenhan Xiong $* \S \quad$ Tian Gao ${ }^{*} \dagger$ \\ ${ }^{\dagger}$ IBM Research $\quad \ddagger$ Umass Amherst $\quad \S$ UC Santa Barbara
}

\begin{abstract}
General Question Answering (QA) systems over texts require the multi-hop reasoning capability, i.e. the ability to reason with information collected from multiple passages to derive the answer. In this paper we conduct a systematic analysis to assess such an ability of various existing models proposed for multi-hop QA tasks. Specifically, our analysis investigates that whether providing the full reasoning chain of multiple passages, instead of just one final passage where the answer appears, could improve the performance of the existing QA models. Surprisingly, when using the additional evidence passages, the improvements of all the existing multi-hop reading approaches are rather limited, with the highest error reduction of $5.8 \%$ on F1 (corresponding to $1.3 \%$ absolute improvement) from the BERT model.

To better understand whether the reasoning chains could indeed help find correct answers, we further develop a co-matchingbased method that leads to $13.1 \%$ error reduction with passage chains when applied to two of our base readers (including BERT). Our results demonstrate the existence of the potential improvement using explicit multi-hop reasoning and the necessity to develop models with better reasoning abilities. ${ }^{1}$
\end{abstract}

\section{Introduction}

More recent development of QA systems (Song et al., 2018; De Cao et al., 2018; Zhong et al., 2019) has started to focus on multi-hop reasoning on text passages, aiming to propose more sophisticated models beyond the shallow matching between questions and answers. Multi-hop reasoning requires the ability to gather information from multiple different passages to correctly answer the question, and generally the task

\footnotetext{
${ }^{*}$ Equal contributions.

${ }^{1}$ Code and data released at https://github.com/ helloeve/bert-co-matching.
}

would be unsolvable by using only similarities between the question and answer. Recent multihop QA datasets, such as WikiHop (Welbl et al., 2018), ComplexWebQuestions (Talmor and Berant, 2018), and HotpotQA (Yang et al., 2018), have accelerated the rapid progress of QA models for multi-hop reasoning problems.

There have been several reading comprehension models proposed to address the problem. Some methods (Yang et al., 2018; Zhong et al., 2019) rely on cross-attention among the question and evidence passages. BERT (Devlin et al., 2018) is one successful model of such an approach. Moreover, a substantial amount of query reformulation approaches (Weston et al., 2014; Wu et al., 2016; Shen et al., 2017; Das et al., 2019) have been proposed. Most of these methods adopt a soft version of reformulation, i.e. modifying the question embeddings based on the attention computed from each reasoning step. Similarly, some hard query reformulation approaches (Buck et al., 2018) propose to rewrite the question in the original language space. These methods provide more transparency to the reasoning processes. However, their performance usually lags behind their soft counterparts when no supervision on re-writing is available.

This paper aims to investigate the following two questions for multi-hop reasoning QA systems:

Do existing models indeed have the multi-hop reasoning ability? To answer this question, we design a dataset with chains of passages ordered by the ground-truth reasoning path. Then we conduct the comparisons between two settings: (1) training and evaluating the models with the correct ordering of the passage chains (the ordered-oracle setting); (2) training and evaluating the models with only the single passage that contain the answer (the single-oracle setting). We hypothesize that if the dataset indeed requires multi-hop rea- 
soning and if a model could conduct multi-hop reasoning, it should perform significantly better in the first setting. However, we discovered that, for all the existing multi-hop reading comprehension models, the performance improvement with the ordered passages is rather limited, with the highest F1 improvement from BERT as $1.29 \%$.

Is it beneficial to explore the usage of the reasoning chains? To answer this question, we try to find a reader model which could indeed make a better use of the the ordered passage information to improve performance. Inspired by the recent progress on the co-matching approaches for answer option selection (Wang et al., 2018; Zhang et al., 2019), we propose to adopt a similar idea for multi-hop question answering. We extend both the HotpotReader (Yang et al., 2018) and the BERT model (Devlin et al., 2018) with co-matching and observe $3.88 \%$ and $2.91 \% \mathrm{~F} 1$ improvement in the ordered-oracle setting over the single-oracle setting. These results confirm that the utilization of passage chains is important for multi-hop question answering, and there is untapped potential of designing new models that could perform "real" multi-hop reasoning.

\section{Analysis Methods}

The goal of this analysis is to validate each model's multi-hop reasoning ability by a specifically designed dataset with three comprehensive experiment settings.

\subsection{Dataset}

We conduct the analysis over a recently released multihop QA dataset HotpotQA (Yang et al., 2018). We created a new empirical setting based on the HotpotQA distractor setting: for each question-answer pair, two supporting passage are labeled by human annotators that are sufficient for answering the question. We release the data of our analysis setting, to make our results comparable for future works. ${ }^{2}$

There have been several multi-hop QA datasets released, but none of them has the ground truth reasoning chains annotated. The reason we choose HotpotQA is that the provided supporting passages serve as a good start point for identifying the approximately correct reasoning chain of passages, based on the heuristics described below. ${ }^{3}$

\footnotetext{
${ }^{2}$ https://gofile.io/?c=FDsdal.

${ }^{3}$ The HotpotQA also contains a subset of comparison
}

The key idea to recover the reasoning chain is that the chain must end at a passage that contains the answer. Specifically, given a question-answer pair $(q, a)$ and its two supporting passages ${ }^{4} p_{0}$, $p_{1}$. Each passage $p_{i}$ is an abstract paragraph of a Wikipedia page, thus corresponding to a topic entity $e_{i}$ that is the title of the page. To determine the reasoning chain of passages, we have the following steps:

- We first check whether the answer $a$ appears in any of the passages. If there is only one passage $p_{i}$ containing the answer, then we have a reasoning chain with $p_{i}$ as the final link of the chain, i.e., $p_{1-i} \rightarrow p_{i}$.

- If both passages contain $a$, then we use the following rule to determine the order: we check whether topic entity $e_{i}$ appears in $p_{1-i}$. If true, we have the chain $p_{1-i} \rightarrow p_{i}$. If there are still multiple matches, we simply discard the question.

For a chain $p_{i} \rightarrow p_{j}$, we denote the first passage as the context passage and the second as the answer passage.

\subsection{Analytical Method for the Ability of Multi-Hop Reasoning}

Based on the aforementioned dataset, we propose a systematical approach to assess the multi-hop reasoning ability of different QA models. We design three experiment settings for different passage chain compositions.

- Single-Oracle, similar to the conventional QA setting that only the question and answer passage are provided while any context passages are omitted.

- Ordered-Oracle, that the question and the extracted ordered context and answer passages are provided.

- Random, similar to Ordered-Oracle but the passages are randomly ordered.

Based on the three settings, ${ }^{5}$ we conduct the fol-

questions, which aims to select between two options by comparing a property of theirs queried by the question, e.g., Did LostAlone and Guster have the same number of members?. These questions are not typical multi-hop questions by our community from the view of deduction. Therefore in this analysis we focus on non-comparison questions.

${ }^{4}$ This heuristic only works for chains of length 2 . To investigate longer chains, more complex rules are required to deal with noise in distant supervision. Popular datasets generally do not require more than 2 hops to answer questions correctly. For example all the questions in HotpotQA has no more than 2 hops. We thus leave this to future work.

${ }^{5}$ Please note that both the Single-Oracle and the OrderedOracle settings are not valid realizations of the full task since they require a-priori knowledge of the answers. The settings 
lowing analysis that each answers a research question related the multi-hop ability of the reading comprehension models:

First, we evaluate existing models on these settings, to answer the question Q1: whether the existing models have the multi-hop reasoning ability. To answer the question, we mainly look at the gap between Single-Oracle and Ordered-Oracle. A model with strong multi-hop reasoning capacity should have better performance in the OrderedOracle setting as the reasoning path is given.

Second, if the existing methods do not show great improvement when the reasoning paths are given, we will hope to confirm Q2: whether our dataset does not require multi-hop reasoning because of some data biases (see Section 6 for examples and discussions of the biases). It is difficult to directly answer Q2, therefore in our analysis we try to answer a relevant question $\mathbf{Q 2}^{\prime}$ : whether the existing models can be further improved on the same dataset with better reasoning techniques. Obviously, if there exists a technique that does better with the oracle-order information. It shows the reasoning paths can indeed introduce additional information in our settings, therefore the answer to $Q 2$ is likely yes. Therefore our dataset and settings can be used as a criterion for evaluating different models' multi-hop reasoning ability, i.e. used for answering $Q 1$.

\section{Baseline Models}

For all methods, there are three inputs for the model: $q$ represents the question, $p_{1}$ the context passage, and $p_{2}$ the answer passage. Accordingly, the word-level encoded hidden sequences for these three inputs are $H^{q} \in \mathbb{R}^{l \times Q}, H^{p_{1}} \in \mathbb{R}^{l \times P_{1}}$, and $H^{p_{2}} \in \mathbb{R}^{l \times P_{2}}$ respectively.

\subsection{Baseline Models}

Bi-Attention Reader (HotpotReader) One common state-of-the-art QA system is the HotpotReader (Yang et al., 2018) which is reported to benefit from the context passages. The system includes self-attention and bi-attention which are the standard practice in many question answering systems. We take this as one baseline as many other methods (Liu et al., 2017; Xiong et al., 2017) generally have similar model architectures.

BERT Reader Another strong baseline is to use the pre-trained BERT model to encode $q, p_{1}$, are used in this paper only for analysis purpose. and $p_{2}$ all together, expecting the inner-attention mechanism to capture the order information.

Given the fact that BERT could only take one input which contains the question and answer separated by "[SEP]", one straightforward approach to encode all three inputs by concatenating the two passages $p_{1}$ and $p_{2}$ to form the answer text " $q$ [SEP] $p_{1} p_{2}$ ". A more explicit way to introduce the separation of the two passages is to include a learnable boundary token by using the reserved token "[unused0]". Therefore we design another input for BERT as " $q$ [SEP] $p_{1}$ [unused0] $p_{2}$ ". We adopt both approaches for completeness.

\section{Multi-hop Reasoning Approaches}

We seek to extend these two baseline models with two commonly used approaches for multi-hop reasoning, i.e. query-reformulation and co-matching.

\subsection{Query-Reformulation Approach}

Query-reformulation is an idea widely used in many multi-step reasoning QA models (Wu et al., 2016; Shen et al., 2017; Das et al., 2019). The key idea is that after the model reads a paragraph, the question representation should be modified according to the matching results between the question and the paragraph. In this way, when the next paragraph comes, the model could focus on "what is not covered" from the history.

Most of the previous methods represent the question as a single vector so that the reformulation is performed in the embedding space. However, representing a question with a single vector performs badly in our task, which is not surprising since most of the top systems on recent QA leaderboards adopt word-by-word attention mechanisms.

Therefore, to have a fair comparison, we need to extend the existing methods from reformulating single vectors to reformulating the whole hidden state sequences $H^{q}$. To compare the first passage $H^{p_{1}}$ with the question $H^{q}$, we applied the BiAtt function and result in the matching states $\tilde{H}^{q} \in \mathbb{R}^{l \times Q}$, where each $\tilde{H}^{q}[:, i]$ states how the $i$ th word of the question is matched by the passage $p_{1}$. Then we use these matching states to reformu- 
late the $H^{q}$ as follows:

$$
\begin{aligned}
\tilde{H}^{q} & =\operatorname{BiAtt}\left(H^{p_{1}}, H^{q}\right) \\
M^{q} & =\gamma H^{q}+(1-\gamma) \tanh \left(W\left[H^{q}: \tilde{H}^{q}: H^{q}-\tilde{H}^{q}\right]\right) \\
\tilde{H}^{p_{2}} & =\operatorname{BiAtt}\left(M^{q}, H^{p_{2}}\right) \\
M & =\operatorname{BiLSTM}\left(\tilde{H}^{p_{2}}\right) \\
M^{\prime} & =\operatorname{SelfAtt}(M)
\end{aligned}
$$

where $\gamma=\sigma\left(W_{g}\left[\tilde{H}^{q}: H^{q}: H^{q}-\tilde{H}^{q}\right]\right)$ is a gate function. For the reformulation equation of $M^{q}$, we have also tried some other popular options, including only with $M^{q}=\tanh \left(W\left[H^{q}\right.\right.$ : $\left.\left.\tilde{H}^{q}: H^{q}-\tilde{H}^{q}\right]\right), M^{q}=\operatorname{BiLSTM} M\left[\tilde{H}^{q}: H^{q}:\right.$ $\left.H^{q}-\tilde{H}^{q}\right]$ and directly set $M^{q}=\tilde{H}^{q}$. Among them, our gated function achieves the best performance.

\subsection{Co-Matching Approach}

The work from (Wang et al., 2018) proposed a comatching mechanism which is used to jointly encode the question and answer with the context passage. We extend the idea to conduct the multi-hop reasoning in our setup. Specifically, we integrate the co-matching to the baseline readers by firstly applying bi-attention described in Equation 2 on $\left(H^{q}, H^{p_{2}}\right)$, and $\left(H^{p_{1}}, H^{p_{2}}\right)$ using the same set of parameters.

$$
\begin{aligned}
\bar{H}^{q} & =H^{q} G^{q} \\
G^{q} & =\operatorname{SoftMax}\left(\left(W^{g} H^{q}+b^{g} \otimes e_{p_{2}}\right)^{T} H^{p_{2}}\right) \\
\bar{H}^{p_{1}} & =H^{p_{1}} G^{p_{1}} \\
G^{p_{1}} & =\operatorname{SoftMax}\left(\left(W^{g} H^{p_{1}}+b^{g} \otimes e_{p_{2}}\right)^{T} H^{p_{2}}\right)
\end{aligned}
$$

where $W^{g} \in \mathbb{R}^{l \times l}$ and $b^{g} \in \mathbb{R}^{l}$ are learnable parameters and $e_{p_{2}} \in \mathbb{R}^{P_{2}}$ denotes a vector of all $1 \mathrm{~s}$ and it is used to repeat the bias vector into the matrix.

We further concatenate the two output hidden sequences $\bar{H}^{q}$ and $\bar{H}^{p_{1}}$, followed by a BiLSTM model to get the final hidden sequence for answer prediction as shown in Equation 3. The start and end of the answer span is predicted based on $M$.

$$
M=\operatorname{BiLSTM}\left(\left[\bar{H}^{q}: \bar{H}^{p_{1}}\right]\right)
$$

Co-Matching in HotpotReader We follow the above co-matching approach on the HotporReader's output directly.

Co-Matching in BERT One straightforward way to achieve co-matching in BERT is to separately encode the question, the first passage and the second one with BERT, and then apply the above co-matching functions on the output hidden sequence as proposed in (Zhang et al., 2019).

However, as observed in the experiments, we believe the inter-attention mechanism (i.e. cross paragraph attention) could capture the order information in an implicit way. Therefore, we still hope to benefit from the cross passage attention inside BERT, but make it better cooperate with three inputs. After the original encoding from BERT, we apply the co-matching ${ }^{6}$ on the output sequence to explicitly encourage the reasoning path. $H^{q}, H^{p_{1}}$, and $H^{p_{2}}$ could be easily obtained by masking the output sequence according to the original text.

\section{Experiments}

\subsection{Settings}

We trained and evaluated each model for comparison for each setting separately. Following previous work (Yang et al., 2018), we report the exactmatch and F1 score for the answer prediction task.

\subsection{Results}

In Table 1, the original HotpotReader method does not show significant performance improvement when comparing the Single-Oracle setting with the Ordered-Oracle setting. BERT was able to get a small improvement from its inner cross passage attention which introduces some weak reasoning. Surprisingly, overall the context passage in the reasoning path does not inherently contribute to the performance of these methods, which indicates that the models are not learning much multi-hop reasoning as previously thought.

\begin{tabular}{lcccc}
\hline \multirow{2}{*}{ Model } & \multicolumn{2}{c}{ Single-Oracle } & \multicolumn{2}{c}{ Ordered-Oracle } \\
& EM & F1 & EM & F1 \\
\hline HotpotReader & 55.07 & 70.00 & 55.17 & 70.75 \\
Bert & 64.08 & 77.86 & 65.03 & 79.15 \\
\hline
\end{tabular}

Table 1: Baseline results for HotpotReader and BERT

We show our proposed improvements in Table 2 and 3. Compared to the Single-Oracle baseline (HotpotReader), when applying the co-matching mechanism in the Ordered-Oracle setting, there is a significant improvement of $4.38 \%$ in exact match and $4.26 \%$ in F1. The soft query reformulation also improves the performance but not as significantly. In order to confirm that the improvement

\footnotetext{
${ }^{6}$ To follow the original BERT's setup, we also apply the same attention dropout with a probability of 0.9 on the attention scores.
} 
of co-matching does come from the usage of reasoning paths (instead of the higher model capacity), we make another comparison that runs the co-matching model over the Single-Oracle setting. To achieve this, we duplicate the single oracle passage twice as $p_{1}$ and $p_{2}$. Our results show that this method does not give any improvement. Therefore the co-matching method indeed contributes to the performance gain of multi-hop reasoning.

\begin{tabular}{lccc}
\hline \multirow{2}{*}{ Model } & \multirow{2}{*}{ Order } & \multicolumn{2}{c}{ Performance } \\
& & EM & F1 \\
\hline \multirow{2}{*}{ HotpotReader } & Random & 52.23 & 69.80 \\
& Single-Oracle & 55.07 & 70.00 \\
& Ordered-Oracle & 55.17 & 70.75 \\
\hline \multirow{2}{*}{ w/ Query-Reform } & Ordered-Oracle & 56.89 & 71.69 \\
\hline \multirow{2}{*}{ w/ Co-Matching } & Single-Oracle & 55.00 & 70.23 \\
& Ordered-Oracle & $\mathbf{5 9 . 4 5}$ & $\mathbf{7 4 . 2 6}$ \\
\hline
\end{tabular}

Table 2: Results for HotpotReader on 3 oracle settings

BERT achieved promising results even in the Single-Oracle setting which proves its original capacity for QA. The original BERT was improved by $1.23 \%$ in exact match when both context passage and answer passage are provided and separated by an extra token. Nonetheless, the comatching mechanism contributes to an additional $1.66 \%$ exact match improvement which indicates the success of co-matching for reasoning. Comatching result also shows that multi-hop over passage chain contains additional information, and thus multi-hop ability is necessary in our analysis setting.

\begin{tabular}{lccc}
\hline \multirow{2}{*}{ Model } & Order & \multicolumn{2}{c}{ Performance } \\
& & EM & F1 \\
\hline \multirow{2}{*}{ BERT } & Random & 59.18 & 75.27 \\
& Single-Oracle & 64.08 & 77.86 \\
\multirow{2}{*}{ w/ split token } & Ordered-Oracle & 65.03 & 79.15 \\
w/ Co-Matching & Ordered-Oracle & 65.31 & 79.49 \\
\hline
\end{tabular}

Table 3: Results for BERT on 3 oracle settings

Among both approaches, co-matching shows promising performance improvement especially for the well pre-trained BERT model. This proves the co-matching mechanism is able to conduct multi-hop reasoning following the passage chains.

Finally, both models perform worse in the Random setting compared to the Single-Oracle setting, although the Random setting contains sufficient information of the whole reasoning chain.
From the analysis, we find that it is difficult for the models to correctly predict the orders from the randomly-ordered passages. For example, we created a binary classification task to predict which passage is the context passage and which is the answer passage. BERT model gives an accuracy of only $87.43 \%$ on this task. This gives further evidence that the existing models do not have appropriate inductive biases for utilizing the reasoning chains.

Answers to our research questions The above results answer our research questions as follows: (1) in our experimental setting, the reasoning paths are indeed useful, thus multi-hop reasoning is necessary, as there exists a method, i.e., co-matching, that has demonstrated significant improvement; (2) existing reader models usually cannot fully make use of the reasoning paths, indicating their limited reasoning abilities. Among the existing methods, BERT can do slightly better on making use of the reasoning paths. Our new proposed comatching approach improves the reasoning abilities over both the two different base models (HotpotReader and BERT).

\section{Discussion}

Difference from prior work Our work conducts the first analysis of models' behaviors. In comparison, a concurrent analysis work (Min et al., 2019), which is also conducted on HotpotQA, focuses more on the properties of the dataset. For example, (Min et al., 2019) finds that for $80 \%$ of the questions in HotpotQA, humans do not need the full paths of paragraphs to correctly answer some of the questions. One of the major reasons is the bias of factoid questions that look for certain types of entities as answers. For example, a question asking "which sports team" can be directly answered if there is only one sports team mentioned in the documents.

Our analysis focuses on whether the full reasoning paths can help the machine learning models to (1) improve their performance on those $80 \%$ of the questions, as well as (2) cover the left $20 \%$ of questions that indeed require the multi-hop ability. Moreover, compared to the prior analysis, we are the first to analyze the effects of reasoning paths in an explicit way, and construct a dataset for this purpose. 
The effect of data biases on our analysis The aforementioned biases make the full reasoning paths less useful for a large portion of data, therefore making it more challenging for reader models to improve with full reasoning paths.

Because of the data bias, it is critical to verify that the dataset we created can still benefit from the improved reasoning skills. That is why answering $Q 2$ in Section 2.2 is important for the whole analysis. The results in Section 5.2 show that our co-matching methods can indeed benefit from the reasoning paths, confirming the effectiveness of our proposed dataset and settings for the analysis purpose.

Encouraging model design with better evaluation Finally, continued from the previous paragraph, we hope to highlight the problem that the less biased a dataset is, the more likely a model can easily benefit from the availability of reasoning paths. On many existing benchmark datasets that are biased, it is less likely to achieve improvement with specific designs for achieving multi-hop reasoning ability. This makes multi-hop reasoning a less important factor when people design models for these multi-hop QA datasets, if the goal is simply to improve the answer accuracy.

To encourage model design towards real reasoning instead of fitting the data biases, we believe that an improved evaluation is necessary. To this end, one way is certainly to create datasets with fewer biases. While our analysis also suggests the other way: we can keep the biased training data, but created small evaluation datasets with humanlabeled reasoning paths. Then during evaluation, we compute the accuracy of the predicted reasoning paths. This is an extension of the idea of HotpotQA that jointly evaluates the support selection and answer extraction, but with a more explicit focus on the reasoning processes.

\section{Conclusion}

In this paper, we analyze QA models' capability in multi-hop reasoning by assessing if the reasoning chain could help existing multi-hop readers. We observed the general weakness of stat-or-the-art models in multi-hop reasoning and proposed a comatching based method to mitigate. Despite the fact that co-matching is designed to encode only three input sequences to achieve limited multi-hop reasoning, we consider this as the most promising one that demonstrates the concrete reasoning capability and has the potential for real multi-hop reasoning.

\section{Acknowledgments}

We thank the anonymous reviewers for their very valuable comments and suggestions.

\section{References}

Christian Buck, Jannis Bulian, Massimiliano Ciaramita, Wojciech Gajewski, Andrea Gesmundo, Neil Houlsby, and Wei Wang. 2018. Ask the right questions: Active question reformulation with reinforcement learning. In International Conference on Learning Representations.

Rajarshi Das, Shehzaad Dhuliawala, Manzil Zaheer, and Andrew McCallum. 2019. Multi-step retrieverreader interaction for scalable open-domain question answering. In International Conference on Learning Representations.

Nicola De Cao, Wilker Aziz, and Ivan Titov. 2018. Question answering by reasoning across documents with graph convolutional networks. arXiv preprint arXiv:1808.09920.

Jacob Devlin, Ming-Wei Chang, Kenton Lee, and Kristina Toutanova. 2018. Bert: Pre-training of deep bidirectional transformers for language understanding. arXiv preprint arXiv: 1810.04805 .

Xiaodong Liu, Yelong Shen, Kevin Duh, and Jianfeng Gao. 2017. Stochastic answer networks for machine reading comprehension. arXiv preprint arXiv:1712.03556.

Sewon Min, Eric Wallace, Sameer Singh, Matt Gardner, Hannaneh Hajishirzi, and Luke Zettlemoyer. 2019. Compositional questions do not necessitate multi-hop reasoning. arXiv preprint arXiv:1906.02900.

Yelong Shen, Po-Sen Huang, Jianfeng Gao, and Weizhu Chen. 2017. Reasonet: Learning to stop reading in machine comprehension. In Proceedings of the 23rd ACM SIGKDD International Conference on Knowledge Discovery and Data Mining, pages 1047-1055. ACM.

Linfeng Song, Zhiguo Wang, Mo Yu, Yue Zhang, Radu Florian, and Daniel Gildea. 2018. Exploring graph-structured passage representation for multihop reading comprehension with graph neural networks. arXiv preprint arXiv:1809.02040.

Alon Talmor and Jonathan Berant. 2018. Repartitioning of the complexwebquestions dataset. arXiv preprint arXiv:1807.09623.

Shuohang Wang, Mo Yu, Shiyu Chang, and Jing Jiang. 2018. A co-matching model for multichoice reading comprehension. arXiv preprint arXiv:1806.04068. 
Johannes Welbl, Pontus Stenetorp, and Sebastian Riedel. 2018. Constructing datasets for multi-hop reading comprehension across documents. Transactions of the Association of Computational Linguistics, 6:287-302.

Jason Weston, Sumit Chopra, and Antoine Bordes. 2014. Memory networks. arXiv preprint arXiv:1410.3916.

Qi Wu, Peng Wang, Chunhua Shen, Anthony Dick, and Anton van den Hengel. 2016. Ask me anything: Free-form visual question answering based on knowledge from external sources. In Proceedings of the IEEE Conference on Computer Vision and Pattern Recognition, pages 4622-4630.

Caiming Xiong, Victor Zhong, and Richard Socher. 2017. Den+: Mixed objective and deep residual coattention for question answering. arXiv preprint arXiv: 1711.00106 .

Zhilin Yang, Peng Qi, Saizheng Zhang, Yoshua Bengio, William W Cohen, Ruslan Salakhutdinov, and Christopher D Manning. 2018. Hotpotqa: A dataset for diverse, explainable multi-hop question answering. arXiv preprint arXiv:1809.09600.

Shuailiang Zhang, Hai Zhao, Yuwei Wu, Zhuosheng Zhang, Xi Zhou, and Xiang Zhou. 2019. Dual comatching network for multi-choice reading comprehension. arXiv preprint arXiv:1901.09381.

Victor Zhong, Caiming Xiong, Nitish Shirish Keskar, and Richard Socher. 2019. Coarse-grain fine-grain coattention network for multi-evidence question answering. arXiv preprint arXiv:1901.00603. 